\title{
Rules of Thumb for Conservation of Metapopulations Based on a Stochastic Winking-Patch Model
}

\author{
Rampal S. Etienne ${ }^{\star}$ and J. A. P. Heesterbeek ${ }^{\dagger}$ \\ Biometris, P.O. Box 100, 6700 AC Wageningen, The Netherlands
}

Submitted March 31, 2000; Accepted May 18, 2001

ABSTRACT: From a theoretical viewpoint, nature management basically has two options to prolong metapopulation persistence: decreasing local extinction probabilities and increasing colonization probabilities. This article focuses on those options with a stochastic, single-species metapopulation model. We found that for most combinations of local extinction probabilities and colonization probabilities, decreasing the former increases metapopulation extinction time more than does increasing the latter by the same amount. Only for relatively low colonization probabilities is an effort to increase these probabilities more beneficial, but even then, decreasing extinction probabilities does not seem much less effective. Furthermore, we found the following rules of thumb. First, if one focuses on extinction, one should preferably decrease the lowest local extinction probability. Only if the extinction probabilities are (almost) equal should one prioritize decreases in the local extinction probability of the patch with the best direct connections to and from other patches. Second, if one focuses on colonization, one should preferably increase the colonization probability between the patches with the lowest local extinction probability. Only if the local extinction probabilities are (almost) equal should one instead prioritize increases in the highest colonization probability (unless extinction probabilities and colonization probabilities are very low). The rules of thumb have an important common denominator: the local extinction process has a greater bearing on metapopulation extinction time than colonization.

Keywords: colonization, extinction, metapopulation, conservation.

Ever since Levins $(1969,1970)$ presented his well-known metapopulation model, it has been clear that the key processes in single-species metapopulation dynamics are local extinction and (re)colonization. For nature management of single-species metapopulations, this has the important consequence that attempts to prolong metapopulation

\footnotetext{
* E-mail: r.s.etienne@plant.wag-ur.nl.

${ }^{\dagger}$ E-mail: j.a.p.heesterbeek@plant.wag-ur.nl.
}

Am. Nat. 2001. Vol. 158, pp. 389-407. (c) 2001 by The University of Chicago. 0003-0147/2001/15804-0006\$03.00. All rights reserved. persistence can either be directed toward decreasing the probability of local extinction or toward increasing the probability of colonization. The former may be achieved by, for instance, improving habitat quality or size (cf. Klok and de Roos 1998), whereas the latter is often attained by building corridors or stepping stones (Schultz 1998). However, corridors may affect local extinction probability as well, both positively (by the rescue effect; Brown and Kodric-Brown 1977) and negatively (by a leakage or dilution effect; Allen et al. 1992). Likewise, improving habitat quality may have positive and negative effects on the ability to generate colonists or to be colonized (a better-quality patch might attract more immigrants and could produce more colonizers due to higher reproduction [see, e.g., Hanski 1994; Vos et al. 2001], or such a patch might sustain a larger population and thus offer less incentive to disperse). These diverse processes make it difficult to answer the practical question of whether, given a limited budget, improving habitat or building corridors (or perhaps a combination of the two) is the best option for metapopulation management. As a first step toward answering this question, we will investigate the problem on the level of local extinction and colonization probabilities: Will metapopulation persistence benefit most from a small change in colonization probabilities or from an equally small change in local extinction probabilities? We will also study variations to this theme: If one focuses on local extinction probabilities in a network of patches, which patch should receive most attention, and similarly, if one focuses on colonization probabilities between patches, which connection between which patches deserves most attention? The setting of this study is a single-species, stochastic winking-patch (terminology of Verboom et al. 1993) or patchoccupancy (terminology of Gosselin 1998) metapopulation model in discrete or continuous time. With this model, the answers to the above questions are straightforward for special cases in which the values for the colonization and local extinction probabilities are known exactly. However, these probabilities are usually only vaguely known, for example, in terms of "high" and "low." Therefore, this article aims at providing rules of thumb, in the 
spirit of Wilson and Willis (1975) and Frank and Wissel (1998), rather than precise answers to the questions above.

\section{The Model}

\section{Discrete Time}

Consider a single-species metapopulation distributed over $n$ patches that can be either occupied or empty. Assume that there is a discrete phase in which local population dynamics take place but no dispersal. After this "extinction phase" there is a "colonization phase." This separation of phases has been suggested by several authors (Akçakaya and Ginzburg 1991; Hansson 1991; Sabelis et al. 1991; Burgman et al. 1993). During the extinction phase, the population in each occupied patch $i$ has an extinction probability $e_{i}$, and during the colonization phase, dispersers from each occupied patch $i$ can colonize an empty patch $j$ with colonization probability $c_{i j}$. For the most part in this article, all these probabilities are considered to be independent; that is, we assume that extinctions and colonizations are not correlated. This means, for example, that we do not incorporate the rescue effect (Brown and Kodric-Brown 1977; Etienne 2000). We will also briefly study a model with correlated extinctions; other, probably less important, correlations require detailed models of dispersal and local dynamics that are beyond the scope of this article.

Because every patch is either occupied (denoted by 1 ) or empty (denoted by 0 ), the metapopulation is in any of $2^{n}$ states. For example, for $n=2$, these states are (patch 2 , patch 1$)=(0,0),(0,1),(1,0)$, and $(1,1)$; for $n=3$, these states are (patch 3 , patch 2 , patch 1$)=(0,0,0),(0,0,1)$, $(0,1,0),(0,1,1),(1,0,0),(1,0,1),(1,1,0)$, and $(1,1,1)$. We will order these states lexicographically as in these examples (this is why the order of the patches may seem a bit odd at first) and number them so that, for example, for $n=2$, the states $(0,0),(0,1),(1,0)$, and $(1,1)$ correspond, respectively, to $1,2,3$, and 4 .

With the extinction and colonization probabilities given, we now describe the dynamics of the metapopulation, that is, the changes in its state. We follow Day and Possingham (1995) and Akçakaya and Ginzburg (1991), but see also Gyllenberg and Silvestrov (1994), who present a similar discrete-time model but without separation of extinction and colonization phases. Suppose that $n=2$ and the metapopulation is in state (patch 2, patch 1$)=(0,1)$. From this state, the metapopulation can reach state $(1,1)$ if the first patch does not go extinct and if it subsequently provides colonists to the second patch to make it occupied. These two events occur with probability $\left(1-e_{1}\right) c_{12}$ because we have assumed independence. If we now define $M_{24}$ to be the probability that the metapopulation changes from state $2(\equiv(0,1)$ in the lexicographical ordering) to state $4(\equiv(1,1))$, then $M_{24}=\left(1-e_{1}\right) c_{12}$. Similarly, we can define $M_{i j}$ as the transition probability that the system moves from state $i$ to state $j$. For any pair of states $i$ and $j$, one can calculate the transition probability as above. The $2^{n} \times 2^{n}$ matrix $\mathbf{M}$ thus defined is the Markov transition matrix of the metapopulation system with $n$ patches. For $n=2$, the transition matrix $\mathbf{M}$ is given by

$$
\begin{gathered}
\mathbf{M}= \\
{\left[\begin{array}{llll}
1 & 0 & 0 & 0 \\
e_{1} & \left(1-e_{1}\right)\left(1-c_{12}\right) & 0 & \left(1-e_{1}\right) c_{12} \\
e_{2} & 0 & \left(1-e_{2}\right)\left(1-c_{21}\right) & \left(1-e_{2}\right) c_{21} \\
e_{1} e_{2} & \left(1-e_{1}\right) e_{2}\left(1-c_{12}\right) & \left(1-e_{2}\right) e_{1}\left(1-c_{21}\right) & M_{44}
\end{array}\right],}
\end{gathered}
$$

where

$$
\begin{aligned}
M_{44}= & \left(1-e_{1}\right) e_{2} c_{12}+\left(1-e_{2}\right) e_{1} c_{21} \\
& +\left(1-e_{1}\right)\left(1-e_{2}\right) .
\end{aligned}
$$

If $e_{i}=e$ and $c_{i j}=c$ for all $i$ and $j$, then matrix $\mathbf{M}$ can be simplified to an $(n+1) \times(n+1)$ matrix $\mathbf{P}$, the elements $P_{k l}$ of which are transition probabilities to go from any state with $k$ occupied patches to any state with $l$ occupied patches. They are given by (see app. A)

$$
\begin{aligned}
P_{k l}= & \sum_{i=0}^{\min (k, l)}\left(\begin{array}{l}
k \\
i
\end{array}\right)(1-e)^{i} e^{k-i}\left(\begin{array}{c}
n-i \\
l-i
\end{array}\right) \\
& \times\left[1-(1-c)^{i}\right]^{l-i}\left[(1-c)^{i}\right]^{n-l},
\end{aligned}
$$

in which the terms

$$
\left(\begin{array}{l}
y \\
x
\end{array}\right)
$$

are combinatorials.

For large $n$, the assumption of equal $c$ is very unrealistic. Patches are usually colonized by their neighbors, not by distant populations. We can easily incorporate these thoughts in the model by replacing $(1-c)^{i}$ in equation (3) by $\left(1-c_{s}\right)^{i_{s}}$ where $i_{s}=(i / n) \min (s, n)$, thus allowing a maximum of $s$ occupied patches to contribute (equally) to colonization. (Below, we will use $s=8$ - so we will be concerned with $c_{8}$-which is quite arbitrary but is motivated by the number of neighbors of a patch in a lattice.) The subscript $s$ is added to $c$ for no other reason than to distinguish this adjusted model from the original one. We remark here that this adjusted model assumes that occupied patches do not form clusters but are randomly distributed in space, which is not true for nearest-neighbor 
colonization. If one wants to be strict, one should use the general model at the cost of a much higher dimension or aggregate patches into a single patch ending up with a much lower dimensional metametapopulation.

Day and Possingham (1995) and Akçakaya and Ginzburg (1991) do not describe the case in which extinctions are correlated (Akçakaya and Ginzburg [1991] do incorporate it in their model but do not specify how). In appendix A, we derive the following analogue of equation (3) with extinctions correlated with parameter $\rho=\sigma^{2} /\left(1+\sigma^{2}\right)$ based on an underlying normal distribution:

$$
\begin{aligned}
P_{k l}= & \sum_{i=0}^{\min (k, l)}\left(\begin{array}{c}
k \\
i
\end{array}\right)\left[\int_{-\infty}^{\left(1+\sigma^{2}\right)^{1 / 2} \Phi^{-1}(e)}\right]^{k-i} \\
& \times\left[\int_{\left(1+\sigma^{2}\right)^{1 / 2} \Phi^{-1}(e)}^{\infty}\right] N_{k}[\boldsymbol{y}, \overrightarrow{0}, \mathbf{Y}(\rho)] d \boldsymbol{y} \\
& \times\left(\begin{array}{c}
n-i \\
l-i
\end{array}\right)\left[1-(1-c)^{i}\right]^{l-i}\left[(1-c)^{i}\right]^{n-l},
\end{aligned}
$$

where $N_{k}[y, \overrightarrow{0}, \mathbf{Y}(\rho)]$ is the $k$-dimensional normal distribution of the $k$-dimensional variable $y$ with zero mean and (co)variance matrix $\mathbf{Y}(\rho)=\sigma^{2} \mathbf{J}+\mathbf{I}$, with $\mathbf{J}$ a $k$-by- $k$ matrix with all elements equal to 1 and $\mathbf{I}$ the $k$-by- $k$ identity matrix; $\left(\int_{\alpha}^{\beta}\right)^{i}$ is shorthand for repeating the integration $\int_{\alpha}^{\beta}$ $i$ times.

One can show (see, e.g., Halley and Iwasa 1998) that the second largest eigenvalue $\lambda_{2}$ of the transition matrix ( $\mathbf{M}$ or $\mathbf{P})$ is a measure of the expected extinction time of the metapopulation:

$$
T_{\text {ext }}=\frac{1}{1-\lambda_{2}}
$$

This extinction time is an average over the extinction times of all initial states, where each state is weighed according to the so-called quasi-stationary distribution (Darroch and Seneta 1965; Gilpin and Taylor 1994; Gosselin 1998), which is the probability distribution of states for a system in pseudoequilibrium.

The expected metapopulation extinction time $T_{\text {ext }}$ is the measure of persistence used in this article. Other measures, such as the basic reproduction number $R_{0}$, are also possible and may lead to different conclusions (for comparison of these measures in a general metapopulation setting, see Etienne and Heesterbeek 2000).

\section{Continuous Time}

If instead of a discrete-time Markov process, a continuoustime Markov process is used, then one uses extinction and colonization rates (probabilities per unit of time) instead of probabilities. To keep the model simple and for reasons that become clear later on, we will only study the case where all extinction rates are equal and all colonization rates are equal, and we will denote these rates by $e_{r}$ and $c_{r}$, respectively. Then one can write down the differential equation for the probability $Q_{x}$ of $x$ patches being occupied:

$$
\begin{aligned}
\frac{d Q_{x}}{d t}= & R_{x-1, x} Q_{x-1}+R_{x+1, x} Q_{x+1} \\
& -\left(R_{x, x-1}+R_{x, x+1}\right) Q_{x}
\end{aligned}
$$

where $R_{k, l}$ is the rate of transition from $k$ occupied patches to $l$ occupied patches and where it is assumed that in infinitesimal time steps no more than one extinction or colonization event can occur. In matrix notation,

$$
\frac{d Q}{d t}=\mathbf{Q R},
$$

where $\boldsymbol{Q}$ represents an $(n+1)$-dimensional vector containing the probabilities of $x=0 \ldots n$ patches being occupied and $\mathbf{R}$ is the $(n+1)-$ by- $(n+1)$ transition matrix with elements

$$
R_{k l}=\left\{\begin{array}{ll}
k e_{r} & \text { for } k=l+1 \\
-k e_{r}-k c_{r}(n-k) & \text { for } k=l \\
k c_{r}(n-k) & \text { for } k=l-1 \\
0 & \text { otherwise }
\end{array} .\right.
$$

For example, if $n=2$, then $\mathbf{R}$ is given by

$$
\mathbf{R}=\left[\begin{array}{lll}
0 & 0 & 0 \\
e_{r} & -e_{r}-c_{r} & c_{r} \\
0 & 2 e_{r} & -2 e_{r}
\end{array}\right]
$$

The expected metapopulation extinction time is related to the second largest eigenvalue $\lambda_{2}$ of $\mathbf{R}$ (Keilson 1979; Frank and Wissel 1998):

$$
T_{\mathrm{ext}}=-\frac{1}{\lambda_{2}}
$$

The extinction and colonization rates can be converted to probabilities using 


$$
\begin{aligned}
& e=1-\exp \left(-e_{r}\right), \\
& c=1-\exp \left(-c_{r}\right),
\end{aligned}
$$

because $\exp \left(-e_{r}\right)$ and $\exp \left(-c_{r}\right)$ are the probabilities of a patch not having become extinct and not having colonized after one time step.

\section{Using the Model for Rules of Thumb in Nature Management}

As announced at the beginning of this article, we will focus on several questions that are important for nature management. First we will study whether a metapopulation benefits (in terms of the expected extinction time) most from a small change in colonization probabilities or from an equally small change in local extinction probabilities. We will then ask two more detailed questions. First, if one focuses on local extinction probabilities, extinction of which patch should receive most attention? Second, if one focuses on colonization probabilities, colonization between which pair of patches deserves most attention? The answers to these two questions depend on the particular metapopulation structure; therefore, we study two extreme structures for each question to gain insight in the system, and then we try to generalize to some rules of thumb. We first use the uncorrelated discrete-time model to obtain these answers and regard the continuous-time model and the correlated discrete-time model as test models to see how robust these answers are to changes in model structure. The changes in model structure that we consider are relatively small, so robustness of our results to these changes is definitely not sufficient to conclude overall robustness. Yet models that are very dissimilar in model structure (and hence seem a better test of robustness) are much more difficult to gauge. Therefore, we restrict ourselves to our relatively small deviations in model structure. Robustness to these deviations may not be sufficient, but it is certainly necessary.

\section{Should One Decrease Local Extinction Probability or Increase Colonization Probability?}

We first consider the situation in which all local extinction probabilities are equal $\left(e_{i}=e\right.$ for all $\left.i\right)$ and all colonization probabilities are equal $\left(c_{i j}=c\right.$ for all $i$ and $\left.j\right)$; this means that we can use matrix P. If we decrease the local extinction probability $e$ by a small amount $\Delta e$ or increase the colonization probability $c$ by a small amount $\Delta c$, then we can ask whether decreasing local extinction probability yields a larger metapopulation extinction time than increasing the colonization probability. Because $T_{\text {ext }}(e, c)$ increases monotonically with increasing $c$ when $e$ is fixed and with decreasing $e$ when $c$ is fixed, it suffices to look for the pairs $(e, c)$ for which

$$
-\left.\Delta e \frac{\partial T_{\mathrm{ext}}}{\partial e}\right|_{(e, c)}=\left.\Delta c \frac{\partial T_{\mathrm{ext}}}{\partial c}\right|_{(e, c)} .
$$

These pairs $(e, c)$ then form the boundary in $(e, c)$ space between regions in $(e, c)$ space where $T_{\text {ext }}$ benefits more from a decrease in $e$ and regions where $T_{\text {ext }}$ benefits more from an increase in $c$. It remains to choose appropriate changes in $e$ and $c$, that is, to choose $\Delta e$ and $\Delta c$. We will assume that

$$
\Delta e=\Delta c
$$

and discuss the reasons for this choice and consequences of other choices at the end of this article. This choice allows us simply to compare the derivatives in equation (12).

Numerical calculations gave graphs pictured in figure $1 \mathrm{~A}$. It can be shown analytically (see app. B) that the curves of figure $1 A-1 C$ cross the $(c=0)$-axis at $e=1-1 /(n-1)$. This means that for small $c$ and $e>1-1 /(n-1)$, it is always better to decrease local extinction probability than to increase colonization probability. This can be understood intuitively as follows: if $e$ is close to 1 , a large contribution to the metapopulation extinction time comes from rapid local extinction in all patches before recolonization even gets the opportunity to increase the metapopulation extinction time. Therefore, a change in the colonization probability has only a small influence. When $n$ increases, the probability of local extinction in all patches together decreases, so the effect of recolonization increases, resulting in a higher upper bound for $e$ when $c$ is small.

From figure $1 A$, one can conclude that if nothing is known about $e$ and $c$, the results suggest that one should aim at decreasing $e$ rather than at increasing $c$ because the region in $(e, c)$ space in which increasing $c$ is favored is substantially smaller than the region where decreasing $e$ is preferred. If we know that the colonization probability is (very) low and the extinction probability is smaller than $1-1 /(n-1)$, then increasing $c$ is the better option. The latter addition makes clear that a hasty conclusion that one should increase $c$ when the metapopulation is most extinction prone is not warranted.

To investigate the robustness of these results to model structure, we repeated the analysis with slightly modified models. First, we considered the adjusted model with a maximum of eight patches contributing to colonization. For this model, we get similar results, but the lines of figure $1 A$ lie a bit higher for $n>8$ (but still do not rise above $c_{8}=0.3$ ) and do not appear to go down again for large $n$ but instead seem to become independent of $n$. 


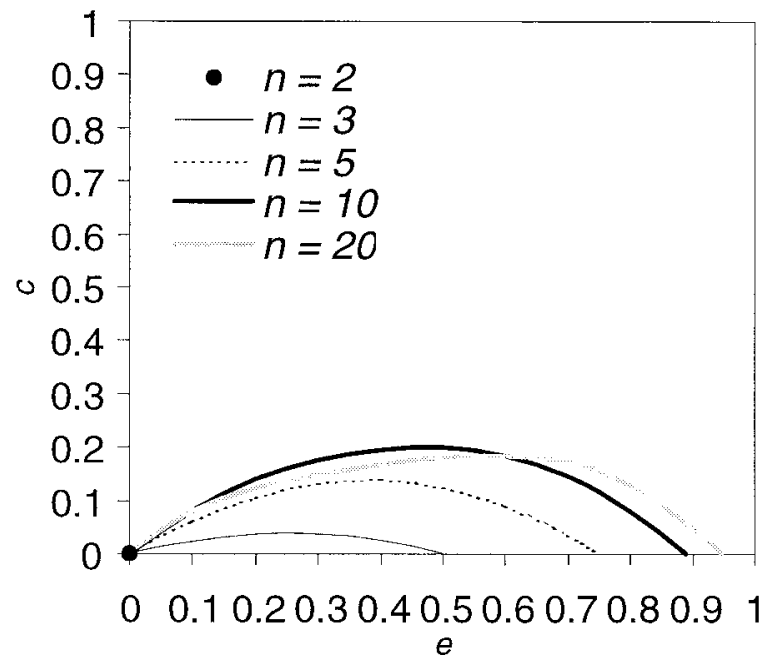

A.

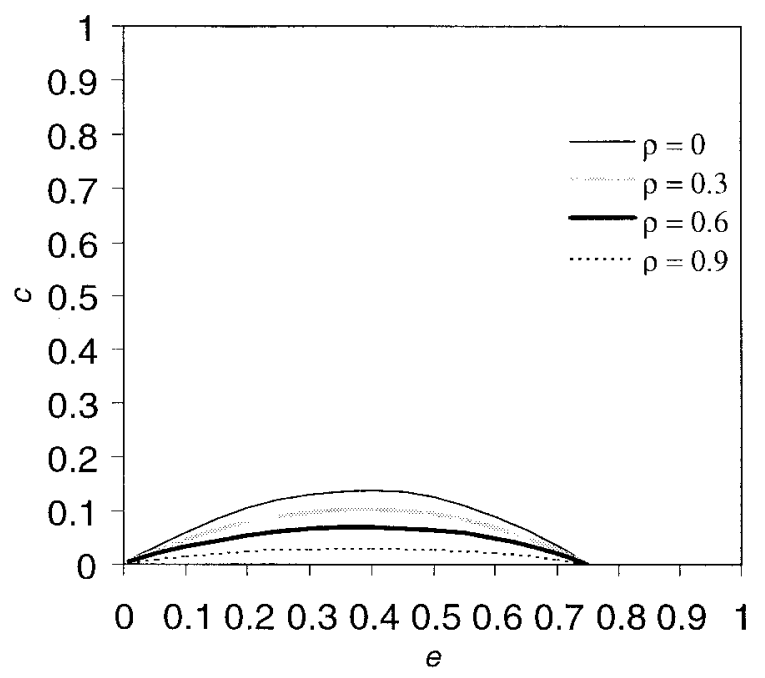

C.

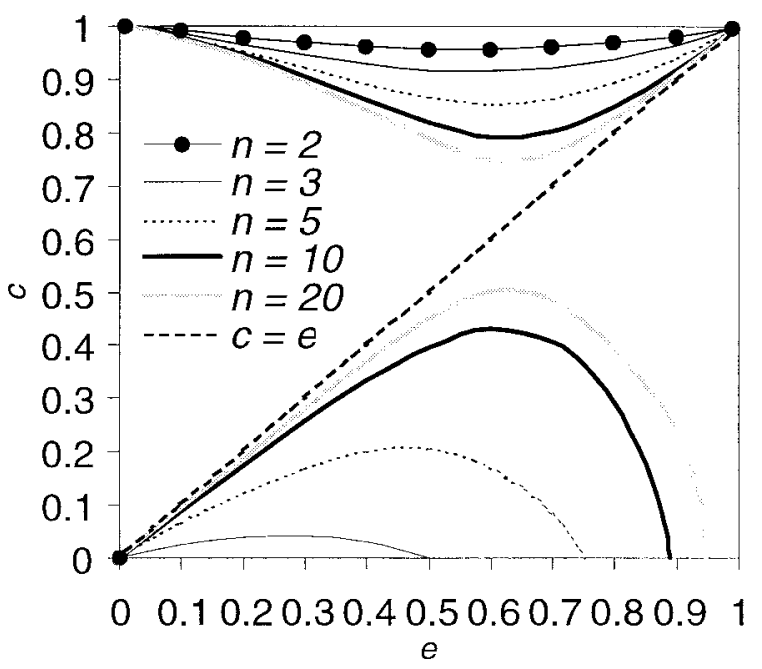

B.

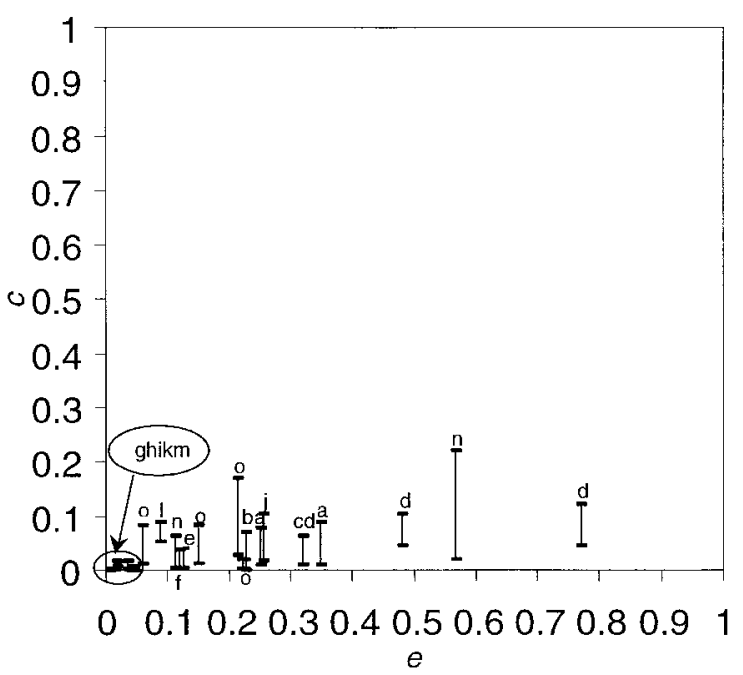

D.

Figure 1: The pairs $(e, c)$ satisfying equations (12) and (13) for several values of $n(A-C)$ and $(e, c)$-pairs estimated from data $(D)$. $A$, The discretetime model. Above the lines, decreasing $e$ increases the metapopulation extinction time more than increasing $c$. Below these lines, the opposite applies. $B$, The continuous-time model. Between the lines, decreasing $e$ increases the metapopulation extinction time more than increasing $c$. Below the lower and above the upper lines, the opposite applies. The line $c=e$ is drawn for convenience; the lines appear to converge to this limit as $n$ approaches infinity. $C$, The discrete-time model for $n=5$ with correlated extinctions for several values of the correlation parameter $\rho$. $D$, Maximum likelihood estimates of the parameter combinations $(e, c)$ and $\left(e, c_{8}\right)$ from turnover data for several metapopulations. The pairs $(e, c)$ and $\left(e, c_{8}\right)$ corresponding to the same metapopulation are connected by a line; its lower end is $(e, c)$, and its upper end is $\left(e, c_{8}\right)$. The models used to estimate $(e, c)$ and $\left(e, c_{8}\right)$ are the (uncorrelated) discrete-time model and its analogue for a limited number of eight patches contributing to colonization. The letters denote the source of the turnover data (with the number of patches in parentheses): $a$, Briers and Warren 2000 (68); $b$, Eber and Brandl 1994, 1996 (513); c, Hanski et al. 1994 (50); d, Lei and Hanski 1998 (50, 22); e, Harrison et al. 1988 (59); f, Hecnar and M’Closkey 1997 (160); g, Hill et al. 1996 (69); h, Kindvall and Ahlén 1992 (110); i, Morrison 1998 (129); j, Nürnberger 1996 (51); k, Smith and Gilpin 1997 (78); l, Sutcliffe et al. 1997 (14); m, Thomas and Harrison 1992 (16, 20); n, Van der Meijden and Van der Veen-Van Wijk 1997 (102, 79); o, Villard et al. 1995 (51). Although the data sets are of different quality, we have not included a measure of the error in the parameter estimates because they are intended for illustrative purposes only. 
Second, we considered the model in continuous time. The results are shown in figure $1 B$. The main difference between figure $1 A$ and $1 B$ is the presence of a second region favoring changes in $c$ that is absent in figure $1 A$. This is caused by the difference in the nature of the underlying models. When $c$ is close to 1 , colonization is almost certain, so all patches are almost always occupied. Therefore, metapopulation extinction can almost only come about if extinctions occur in all patches within a very short time interval so that recolonization cannot take place. In the discrete-time model, there is a finite period in which all patches can go extinct together, so decreasing $e$ always has some impact, even for the extreme case $c=1$. In the continuous-time model, simultaneous extinctions were assumed to be impossible, so metapopulation extinction can occur only by subsequent extinctions of all patches without recolonizations in between. Hence, if $c=1$, metapopulation extinction is impossible, so decreasing $e$ has no effect whatsoever. This makes it plausible that for values of $c$ only a little smaller than 1 , decreasing $e$ has hardly any influence, while increasing $c$ brings the system even closer to the situation where a change in $e$ has no effect. Another difference between figure $1 A$ and $1 B$ is that in figure $1 A$, for fixed $e$, the corresponding value of $c$ initially increases as $n$ increases but then starts to decrease at some value of $n$ (which depends on $e$ ), whereas in figure $1 B$, the values of $c$ below the line $c=e$ keep increasing with $n$; they seem to approach the line $c=e$. This elevation of the lines occurs because increasing $c$ changes colonization probabilities for all $n(n-1)$ pairs of patches, although decreasing $e$ only changes extinction probabilities of $n$ patches, a difference of a factor $n-1$ in favor of increasing $c$. In the discrete-time model, there is an opposing force in favor of decreasing $e$, which dominates for large $n$ : the probability of local extinction always benefits from decreasing $e$, whereas the probability of a patch being colonized does not gain much from increasing $c$ when $n$ is quite large. This opposing force is (almost) absent in the continuoustime model. The continuous-time model thus yields results similar to the discrete-time model: decreasing $e$ is the preferred strategy unless $c$ is small and $e<1-1 /(n-1)$ (see app. B). However, the continuous-time model favors increasing $c$ when $n$ becomes large $(n>30)$ or when $c$ is quite large.

Third, to gain understanding of possible effects of correlated extinctions on the results, we repeated the analysis for $n=5$ using the discrete-time model with correlated extinctions (eq. [4]) for several values of the correlation parameter $\rho$. The resulting figure $1 C$ shows that correlation between extinctions makes the region in $(e, c)$ space in which increasing $c$ is favored become smaller with increasing $\rho$. More generally, we expect that correlation strengthens the effect of decreasing the local extinction probability.
Finally, we examined what happens when we drop the assumption of equal $e$ and equal $c$. We considered 1,000 sets of randomly chosen $e_{i}$ and $c_{i j}$ (i.e., drawn uniformly from one of several choices of intervals, e.g., $0<e_{i}, c_{i j}<$ 1 , or $\left.0<e_{i}<1-1 /[n-1], 0<c_{i j}<0.2\right)$ for the case $n=5$ and determined, in the spirit of equation (12), whether or not

$$
-\left.\sum_{i=1}^{n} \Delta e_{i} \frac{\partial T_{\mathrm{ext}}}{\partial e_{i}}\right|_{(e, c)}>\left.\sum_{i, j \neq i}^{n} \Delta c_{i j} \frac{\partial T_{\mathrm{ext}}}{\partial c_{i j}}\right|_{(e, c)}
$$

with

$$
\Delta e_{i}=\Delta c_{i j}
$$

and $(\boldsymbol{e}, \boldsymbol{c})$ denoting the entire set of extinction and colonization probabilities. This could be interpreted as a comparison between changes in patch habitat quality and matrix (between-patch) habitat quality. The results show that the left-hand side of equation (14) is almost always greater than the right-hand side, even for $0<e_{i}<0.5,0<c_{i j}<$ 0.2 (fig. 2). Only if the $e_{i}$ do not differ much (e.g., all equal) and the $c_{i j}$ are small (e.g., $0<c_{i j}<0.2$ ) is the righthand side greater for a substantial number of the 1,000 sets $(58.9 \%$ in the example; see also fig. $2 D)$. But, more important, when extinction changes are favored, it is by a factor of up to 500, while when colonization is more important, it is by only a twofold difference at most.

Until now, we have compared decreasing $e$ and increas-

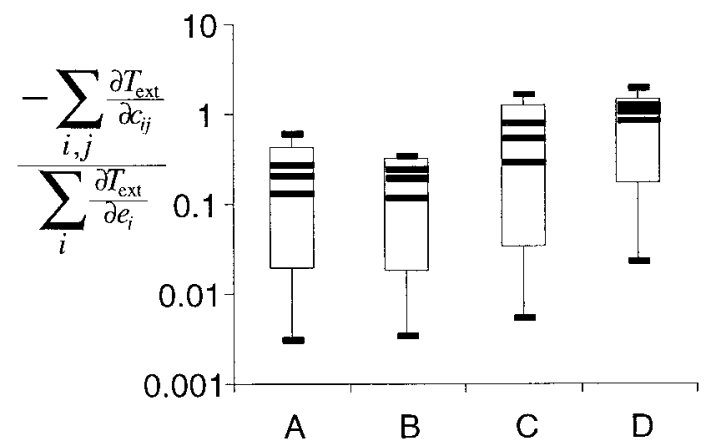

Figure 2: Boxplots of the sensitivity to colonization relative to the sensitivity to extinction $\left(\sum_{i, j}^{n}\left[\partial T_{\text {ext }} / \partial c_{i j}\right] / \sum_{i}^{n}\left[\partial T_{\text {ext }} / \partial e_{i}\right]\right)$ for 1,000 sets of randomly chosen $e_{i}$ and $c_{i j}$ values $\left(c_{i j} \neq c_{j i}\right)$ with $(A) 0<e_{i}, c_{i j}<1$; (B) $0<$ $e_{i}<1,0.4<c_{i j}<0.6$; (C) $0<e_{i}<0.5,0<c_{i j}<0.2$; (D) $0<e_{i}=e<1,0<$ $c_{i j}<0.2$. Other intervals yielded similar plots. The lower bound of the box is the 2.5th percentile ( 25 sets have a lower value of sensitivity to colonization than this), the upper bound is the 97.5th percentile, and the lines in the box denote the 25th, 50th, and 75th percentiles. The ends of the vertical lines above and below the box are the maximum and minimum. 
ing $c$ on the metapopulation level: all probabilities participated. We can also make the comparison on a local level; for example, comparing decreasing $e_{i}$ with increasing $\sum_{j}\left(c_{i j}+c_{j i}\right)$, that is, a comparison between lowering extinction risk and raising disperser input and output of a patch. We did this in the 1,000 sets for the $n=5$ case we have just discussed and found that the results are qualitatively the same: decreasing the extinction probability is far more effective than increasing the sum of colonization probabilities for any patch.

First Rule of Thumb. From all these results we induce the following rule of thumb: To increase metapopulation extinction time, decreasing local extinction probability is preferred over increasing colonization probability; this is strengthened if extinctions are correlated. Only if the colonization probabilities are (very) low and the extinction probabilities are almost equal and smaller than $1-$ $1 /(n-1)$ or if the colonization probability is high and colonization occurs continually is increasing the colonization probability preferred.

Thus, if we have no knowledge about the extinction and colonization probabilities of a particular metapopulation we need to manage, the rule of thumb proposes that we decrease the extinction probability. This reasoning is based on the assumption that, if any further knowledge is lacking, all combinations of $e$ and $c$ are equally likely. In figure $1 D$, we have plotted the most likely combinations of $(e$, $c)$ and $\left(e, c_{8}\right)$ of several species estimated from data on the number of extinctions and colonizations using, respectively, equation (3) and equation (3) with $(1-c)^{i}$ replaced by $\left(1-c_{8}\right)^{i_{8}}$. It is evident that values of $e$ smaller than 0.5 and $\mathcal{c}_{8}$ (which is more realistic than $\mathcal{c}$, particularly in large metapopulations) smaller than 0.2 are most common, so it seems that the exception mentioned in the rule applies to these metapopulations. However, while it is clear that metapopulation management should (also) be based on data, we caution that calculations from data should not be followed blindly (see "Discussion").

\section{In Which Patch Should the Local Extinction Probability Be Decreased?}

To address this question, we consider two extreme situations in both of which we look for that patch $i$ for which a small decrease $\Delta e_{i}$ in the local extinction probability results in the largest increase in the metapopulation extinction time; that is, we look for that patch $i$ for which

$$
-\Delta e_{i} \frac{\partial T_{\mathrm{ext}}}{\partial e_{i}}
$$

is largest. In the first situation, all colonization probabilities are equal $\left(c_{i j}=c\right.$ for all $i$ and $\left.j\right)$, but the local ex- tinction probabilities $e_{i}$ are allowed to differ. In the second situation, all local extinction probabilities are equal ( $e_{i}=e$ for all $i$ ), but the colonization probabilities $c_{i j}$ are allowed to differ. We make the assumption, analogous to equation (13), that $\Delta e_{i}$ is the same for all $i$, so in fact, we look for the patch $i$ for which $\partial T_{\text {ext }} / \partial e_{i}$ is largest.

In the first situation $\left(c_{i j}=c\right.$ for all $i$ and $\left.j\right)$, we numerically calculated $\partial T_{\text {ext }} / \partial e_{i}$ for 1,000 sets of randomly chosen $e_{i}\left(0<e_{i}<1\right)$ for various values of $c$ and $n$. Since calculations for large $n$ are very time consuming, we restricted ourselves to $n \leq 7$. The results indicate that the metapopulation extinction time is mostly affected by a change in the smallest $e_{i}$. In all sets, this result was found.

This result can be understood as follows. We have for the discrete-time model

$$
\begin{gathered}
T_{\mathrm{ext}}(c=0)=\frac{1}{\min _{j}\left(e_{j}\right)}, \\
\left.\frac{\partial T_{\mathrm{ext}}}{\partial e_{i}}\right|_{c=0}= \begin{cases}-T_{\mathrm{ext}}^{2}(c=0) & \text { if patch } i \text { has the smallest } e_{i} \\
0 & \text { for all other patches }\end{cases} \\
T_{\mathrm{ext}}(c=1)=\frac{1}{\prod_{j=1}^{n} e_{j}}, \\
\left.\frac{\partial T_{\mathrm{ext}}}{\partial e_{i}}\right|_{c=1}=-\frac{1}{e_{i}} T_{\mathrm{ext}}(c=1) .
\end{gathered}
$$

In both cases, we find that $\partial T_{\text {ext }} / \partial e_{i}$ is largest in absolute value for the patch $i$ with the smallest value of $e_{i}$. Because $T_{\text {ext }}$ is monotone in $e_{i}$ and $c$, it is not to be expected that this will be different for intermediate values of $c$.

In the second situation ( $e_{i}=e$ for all $\left.i\right)$, we numerically computed $\partial T_{\text {ext }} / \partial e_{i}$ for 1,000 sets of randomly chosen $c_{i j}$ $\left(0<c_{i j}<1\right)$ for various values of $e$ and $n \leq 7$. The results indicate that, in general, the largest influence on metapopulation extinction time is obtained by a change in the $e_{i}$ of the best-connected patch, that is, a change in the local extinction probability of that patch $i$ for which $\sum_{j}\left(c_{i j}+\right.$ $\left.c_{j i}\right)$ is largest. This means that for $n=2$, there would be no preference between the patches, which was indeed the case in the calculations. To give some idea of how well this criterion works, the percentage of sets for which this criterion indeed picked the right patch (the patch for which decreasing the local extinction probability prolongs metapopulation persistence most), exceeded $70 \%$ for all values of $n$ we studied $(n \leq 7)$. Moreover, decreasing the extinction probability of the worst connected patch is almost never the best option to prolong metapopulation persistence. No significant trend for different values of $e$ was observed nor was there any significant change if values for $c_{i j}$ were restricted to a smaller interval than between 0 
and 1 with a different mean than 0.5 (we chose $0<c_{i j}<$ $0.2,0.8<c_{i j}<1$, and $0.4<c_{i j}<0.6$ for comparison). However, there is a significant drop in the percentage for which the criterion picks the right patch when $n$ increases, but this is simply due to the presence of more patches competing for the position of best-connected patch. If we allow a patch to be the right patch if it belongs to the top $20 \%$, say, of best-connected patches, then there is no such decrease. When we set $c_{i j}=c_{j i}$, the percentage of correct picks became even higher than with $c_{i j} \neq c_{j i}$.

Thus, the results suggest that to increase metapopulation extinction time, one should decrease the lowest local extinction probability or the local extinction probability of the best-connected patch, that is, the patch $i$ for which $\sum_{j}\left(c_{i j}+c_{j i}\right)$ is largest. Evidently, in a situation where all parameters may differ, the patch with the lowest extinction probability will generally not be the best-connected patch. To study the trade-off between "lowest extinction probability" and "best connected," we considered the case $n=5$ and calculated $\partial T_{\text {ext }} / \partial e_{i}$ for 1,000 sets of randomly chosen $e_{i}$ and $c_{i j}$ for several intervals of these probabilities $\left(0<e_{i}<1,0<c_{i j}<1 ; 0<e_{i}<1,0<c_{i j}<0.2 ; 0<e_{i}<0.5\right.$, $\left.0<c_{i j}<1 ; 0<e_{i}<0.5, \quad 0<c_{i j}<0.2\right)$ where we chose $c_{i j}=c_{j i}$ for simplicity, thereby getting the strongest effect of $\sum_{j}\left(c_{i j}+c_{j i}\right)$. We found that "lowest extinction probability" very strongly dominates over "best connected," that is, largest $\sum_{j}\left(c_{i j}+c_{j i}\right)$. In the four above-mentioned parameter intervals, we observed that for $94.6 \%, 98.2 \%$, $95.2 \%$, and $96.5 \%$ of the sets, decreasing the $e_{i}$ of the patch with the lowest $e_{i}$ gave the largest $\partial T_{\text {ext }} / \partial e_{i}$. For the patch with the largest $\sum_{j}\left(c_{i j}+c_{j i}\right)$, these percentages were $21.4 \%$, $18.9 \%, 21.2 \%$, and $20.0 \%$, which is insignificant because for $n=5$, a total indifference to $\sum_{j}\left(c_{i j}+c_{j i}\right)$ would give $20 \%$. Figure 3 gives an impression of the relative differ- ences in $\partial T_{\text {ext }} / \partial e_{i}$ for the patch with the lowest extinction probability, the second lowest extinction probability, and so on, and for the patch with the best connections, the second best connections, and so on. We can see from figure $3 A$ that sometimes choosing the patch with the lowest extinction probability is not the optimal choice, but the difference with the real optimum is always relatively small, whereas choosing a patch with the second lowest extinction probability may already differ up to a factor of 1,000 with the real optimum. In figure $3 B$, we indeed observe that choosing the best-connected patch does not guarantee at all that one is even close to the real optimum.

Second Rule of Thumb. Summarizing, we find the following rule of thumb, given that we focus on extinction: To increase metapopulation extinction time, one should preferably decrease the lowest local extinction probability. Only if the extinction probabilities are (almost) equal should one preferably decrease the local extinction probability of the best-connected patch, that is, the patch $i$ for which $\sum_{j}\left(c_{i j}+c_{j i}\right)$ is largest.

\section{Between Which Patches Should the Colonization Probability Be Increased?}

To address this question, we consider the same two extreme situations as above, but now we look for that pair of patches $i$ and $j$ for which a small increase $\Delta c_{i j}$ in the colonization probability results in the largest increase in the metapopulation extinction time; that is, we look for that combination of patches $i$ and $j$ for which

$$
\Delta c_{i j} \frac{\partial T_{\mathrm{ext}}}{\partial c_{i j}}
$$

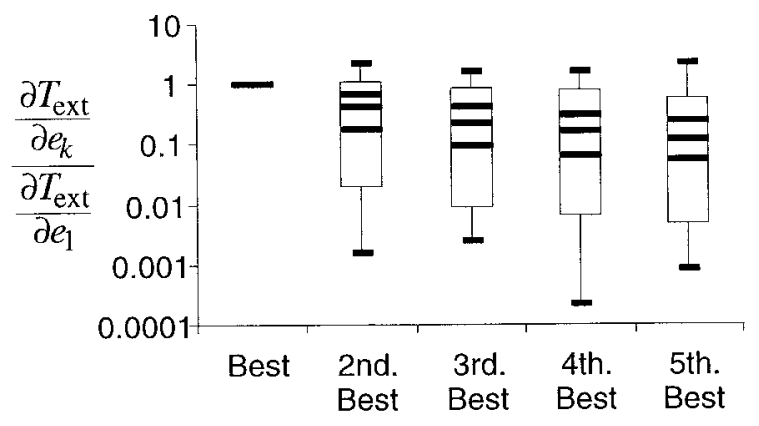

A. $k$ th. best means $k$ th. smallest $e_{i}$

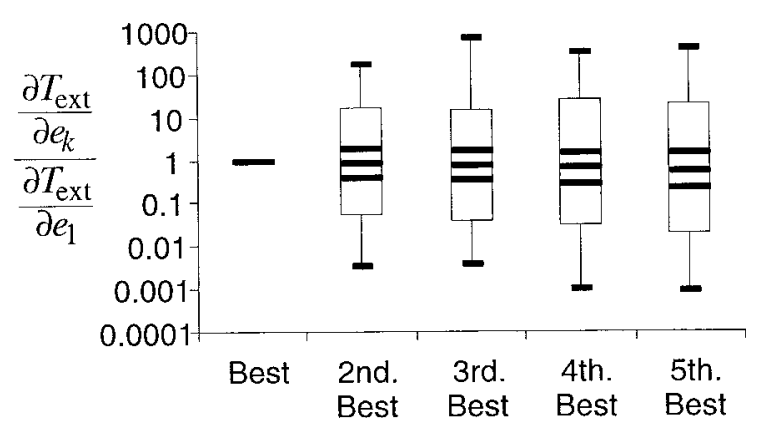

B. $k$ th. best means $k$ th. largest $\sum_{j}\left(c_{i j}+c_{j i}\right)$

Figure 3: Boxplots of $\left(\partial T_{\text {ext }} / \partial e_{k}\right) /\left(\partial T_{\text {ext }} / \partial e_{1}\right)$ with $k$ denoting the $k$ th best patch, $k$ th best meaning $(A) k$ th lowest extinction probability or $(B) k$ th largest sum of colonization probabilities, for the 1,000 sets of randomly chosen $e_{i}$ and $c_{i j}$ values. Here, $0<e_{i} c_{i j}=c_{j i}<1$, but boxplots for the other intervals of the parameters mentioned in the text look very similar. 


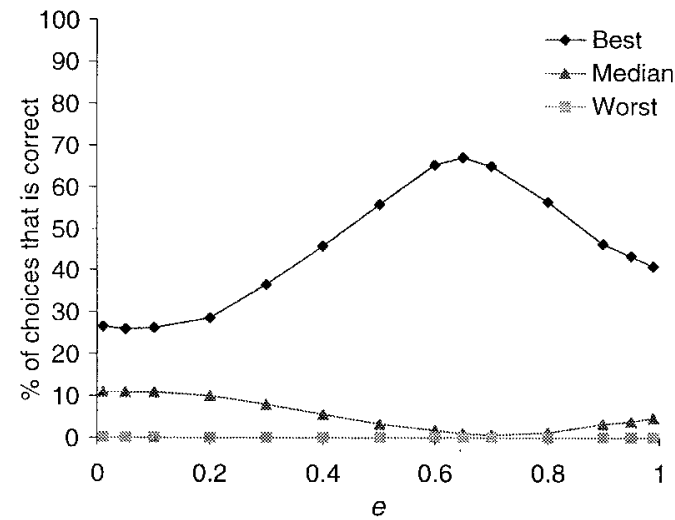

A.

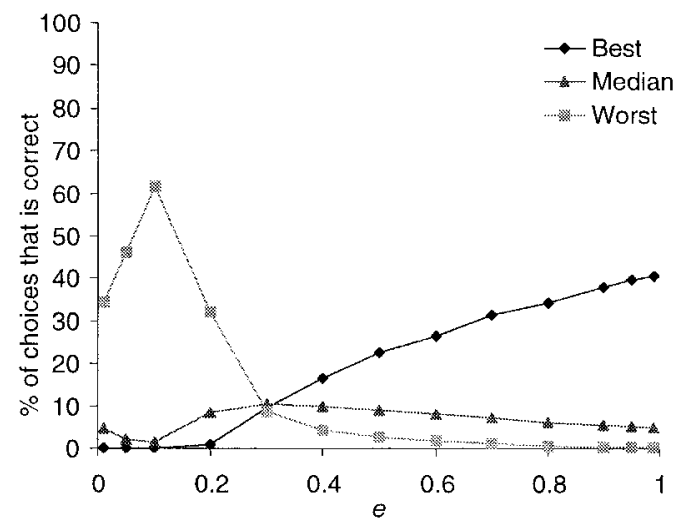

C.

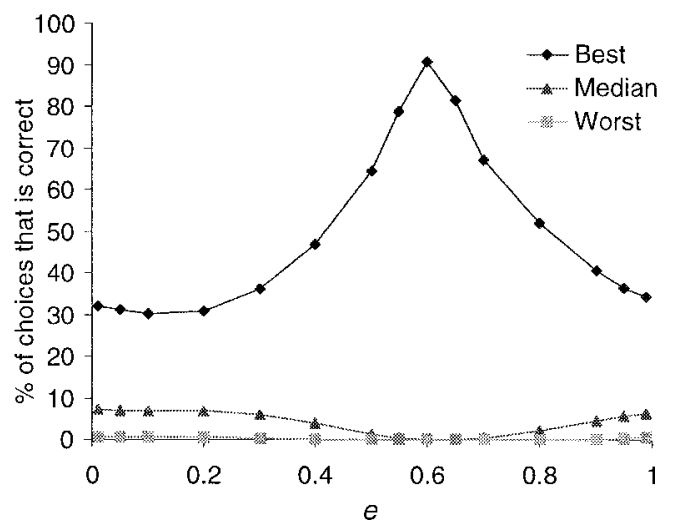

B.

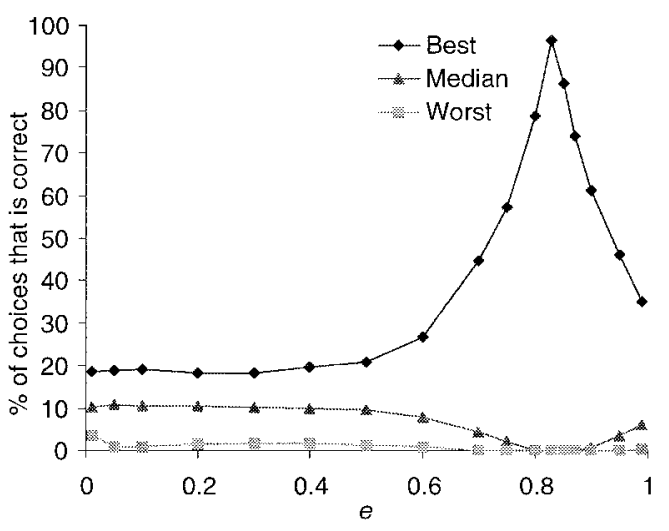

D.

Figure 4: The percentage of cases (out of 1,000) in which increasing the highest (best), median, and lowest (worst) colonization probability $c_{i j}$ has the largest impact on the metapopulation extinction time for $n=5$, several $e$, and all $c_{i j}$ randomly chosen in the range $(A) 0<c_{i j}<1,(B) 0.4<$ $c_{i j}<0.6,(C) 0<c_{i j}<0.2$, and $(D) 0.8<c_{i j}<1$. For simplicity, $c_{i j}=c_{j i}$ is chosen.

is largest. We make the assumption, analogous to equations (13) and (16), that $\Delta c_{i j}$ is the same for all combinations of $i$ and $j$, so in effect, we look for the patches $i$ and $j$ for which $\partial T_{\text {ext }} / \partial c_{i j}$ is largest.

In the first situation $\left(c_{i j}=c\right.$ for all $i$ and $\left.j\right)$, we numerically calculated $\partial T_{\text {ext }} / \partial c_{i j}$ for 1,000 sets of randomly chosen $e_{i}$ for various values of $c$ and $n \leq 7$. The results suggest that one should always "put one's money on" increasing that $c_{i j}$ between the patches $i$ and $j$ with the lowest extinction probability, that is, the $i$ and $j$ for which $e_{i}+$ $e_{j}$ (or $e_{i} e_{j}$ ) is minimal. Moreover, no difference between $\partial T_{\text {ext }} / \partial c_{i j}$ and $\partial T_{\text {ext }} / \partial c_{j i}$ was observed. In all sets, this result was found.

In the second situation $\left(e_{i}=e\right.$ for all $\left.i\right)$, we numerically computed $\partial T_{\text {ext }} / \partial e_{i}$ for 1,000 sets of randomly chosen $c_{i j}$ for various values of $e$ and $n \leq 7$. For these computations, matters are more complicated than above. To simplify somewhat, we chose $c_{i j}=c_{j i}$. Then, in general, an increase in the largest $c_{i j}$ has the greatest impact on the metapopulation extinction time unless the $c_{i j}$ are all very small and $e$ is small (fig. 4). In the latter case, the smallest $c_{i j}$ has the greatest impact (fig. 4C). Furthermore, as $e$ increases, the percentage of sets in which the largest $c_{i j}$ is indeed the one with the largest impact on $T_{\text {ext }}$ increases significantly up to a certain value of $e$, after which it decreases. This final decrease can be understood following a line of reasoning used earlier. When $e$ is very large, metapopulation extinction occurs before colonization even gets the opportunity to prolong metapopulation longevity; therefore, there is no strong preference for increasing any particular colonization probability as $e$ becomes very high.

Thus, the results suggest that to increase metapopulation extinction time, one should increase either the colonization probability between the patches with the lowest extinction probability or the highest colonization probability (unless extinction probabilities and colonization proba- 
bilities are very low). Naturally, in a situation where all parameters differ, the colonization probability between the patches with the lowest extinction probability will generally not be the highest. To study the trade-off between "between the lowest extinction probabilities" and "highest colonization probability," we considered, just as for the previous question, the case $n=5$ and calculated $\partial T_{\text {ext }} / \partial c_{i j}$ for 1,000 sets of randomly chosen $e_{i}$ and $c_{i j}$ for several intervals of these probabilities $\left(0<e_{i}<1,0<c_{i j}<1 ; 0<e_{i}<1\right.$, $0<c_{i j}<0.2 ; 0<e_{i}<0.5,0<c_{i j}<1 ; 0<e_{i}<0.5,0<c_{i j}<$ 0.2 ), where we chose $c_{i j}=c_{j i}$ for simplicity. We found that "between the lowest extinction probabilities" strongly dominates over "highest colonization probability." In the four above-mentioned parameter intervals, we observed that for $83.0 \%, 90.0 \%, 56.0 \%$, and $92.2 \%$ of the sets, increasing the $c_{i j}$ of the connection between the patches with the lowest $e_{i}$ gave the largest $\partial T_{\text {ext }} / \partial c_{i j}$. For the connection with the largest $c_{i j}$, these percentages were $14.3 \%, 12.4 \%$, $21.1 \%$, and $9.8 \%$, which is not significant. Figure 5 gives an impression of the relative differences in $\partial T_{\text {ext }} / \partial c_{i j}$ for the connection between the two lowest extinction prob- abilities, between the two second lowest extinction probabilities, and so on, and for the patch with the highest colonization probability, the second highest colonization probability, and so on.

Third Rule of Thumb. To recapitulate, the following rule of thumb can be formulated, given that we focus on colonization: To increase metapopulation extinction time one should preferably increase the colonization probability between the patches with the lowest extinction probability. Only if the extinction probabilities are (almost) equal should one preferably increase the highest colonization probability (unless extinction probabilities and colonization probabilities are very low).

\section{Discussion}

The results of this article lead to three rules of thumb. First, for most combinations of the local extinction probability $e$ and the colonization probability $c$, a decrease in $e$ will increase the metapopulation extinction time more than would a comparable increase in $c$. This suggests that,

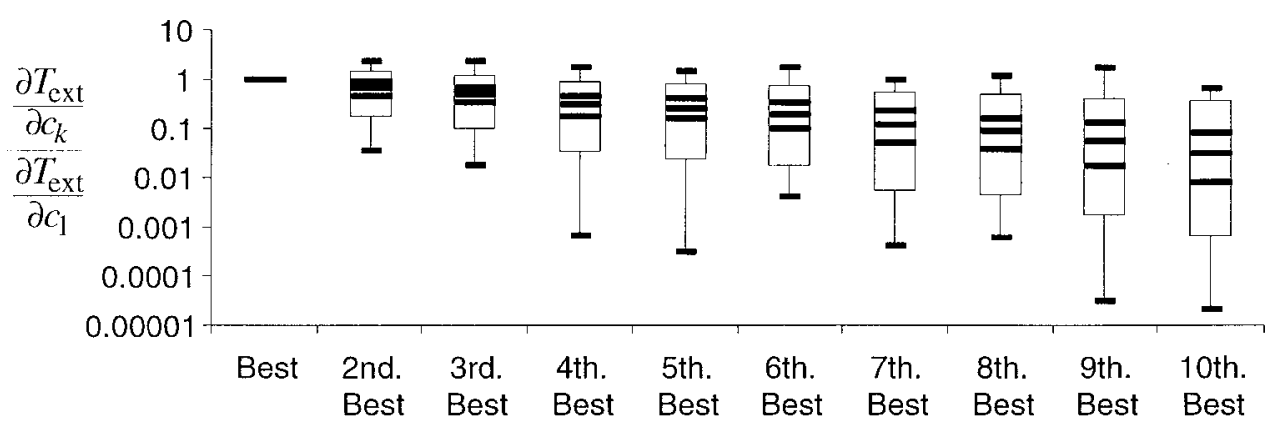

A. $k$ th. best means $k$ th. smallest $e_{i}+e_{j}$

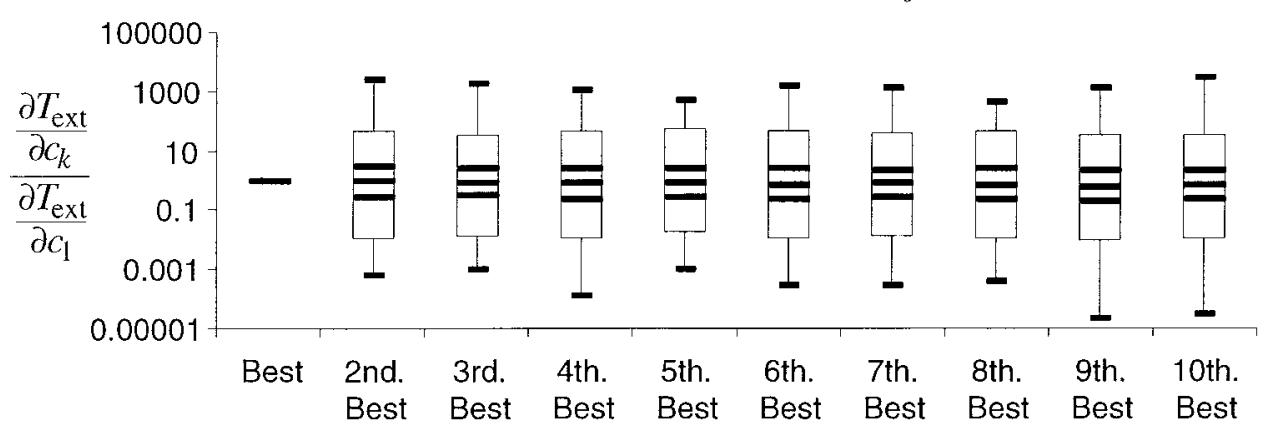

B. $k$ th. best means $k$ th. largest $c_{i j}$

Figure 5: Boxplots of $\left(\partial T_{\text {ext }} / \partial c_{k}\right) /\left(\partial T_{\text {ext }} / \partial c_{1}\right)$ with $k$ denoting the $k$ th best, $k$ th best meaning $(A)$ the $c_{i j}$ with the $k$ th smallest value of $e_{i}+e_{j}$ or $(B)$ $k$ th highest colonization probability $c_{i j}$, for the 1,000 sets of randomly chosen $e_{i}$ and $c_{i j}$ values. Here, $0<e_{i}, c_{i j}=c_{j i}<1$, but boxplots for the other intervals of the parameters mentioned in the text look very similar. Because $c_{i j}=c_{i j}$ and $\partial T_{\text {ext }} / \partial c_{i j}=\partial T_{\text {ext }} / \partial c_{i j}$, the number of possible values for $k$ equals 10 . 
in general, one should focus on decreasing $e$ rather than on increasing $c$, even more so when extinctions are correlated. This preference for lowering extinction probability is in agreement with general conclusions in the literature (e.g., Goel and Richter-Dyn 1974; Drechsler and Wissel 1998). Second, if one focuses on decreasing local extinction probability, then one should generally select the patch with the lowest local extinction probability; only if the extinction probabilities are (almost) equal should one generally select the patch with the best direct connections in terms of the largest value of $\sum_{j}\left(c_{i j}+c_{j i}\right)$. We expect that the latter criterion works only if indirect connections do not vary much between patches, such that the sum of direct connections are a good proxy for overall connectivity. Third, if one focuses on increasing colonization probability, then one should generally select the colonization probability between the patches with the lowest extinction probability; only if the extinction probabilities are (almost) equal should one select the highest colonization probability, or at least not the lowest.

We stress that these rules of thumb should be treated with caution (as should all rules of thumb). We will discuss some of their limitations below, but first, we want to remark that these rules of thumb show an important similarity that may be more robust than the rules of thumb themselves: extinction appears as a more dominant process than colonization. The main reason for this is presumably that extinction is not only important on a generation basis (i.e., affecting the number of patches colonized by an occupied patch during its lifetime as an occupied patch) but also in real time (see also Goel and Richter-Dyn 1974; Diekmann and Heesterbeek 2000), both of which are important for the expected metapopulation extinction time. In contrast, colonization plays a part only on a generation basis. If an alternative measure of metapopulation persistence is used-such as $R_{0}$ (the mean number of patches colonized by an occupied patch in an otherwise empty environment) and its related, computationally simpler measures of colonization potential (Etienne and Heesterbeek 2000) and metapopulation capacity (Hanski and Ovaskainen 2000), or the mean occupancy-the dominance of extinction might disappear. The measure to be used depends on our management goals and the metapopulation in question. The variable $R_{0}$ (or colonization potential or metapopulation capacity) is a measure of the resilience of the metapopulation after a catastrophe (fire, drought, disease), while patch occupancy stresses abundance, not just presence of a species. We chose the expected time to metapopulation extinction as a measure of persistence because of its easy interpretation and its connection with real time (in which we live), but we remark that it may be fairly useless in specific cases, for example, when catastrophes are likely to occur on a timescale shorter than the metapopulation extinction time. Furthermore, we need to stress that the expected metapopulation extinction time depends on the initial state. We took as our initial state the quasi-stationary state, which was said to represent pseudoequilibrium. Pseudoequilibrium seems to be the most neutral starting point for deriving rules of thumb for metapopulations in general. One may wonder whether mathematical pseudoequilibrium corresponds to the ecological pseudoequilibrium we have in mind, but without a clearly better alternative, the choice seems fair, especially if we remember that only changes in the metapopulation extinction time were studied instead of predicted values for the metapopulation extinction time itself.

From the common denominator in the rules of thumb-local extinction is more important than colonization-it would seem that one could conclude that the metapopulation approach to management is not very effective; it seems better to manage a single extinction-proof patch and regard the other patches as a bonus, that is, a mainland-island setting. Add to this the risks of increasing colonization (e.g., facilitating spread of pathogens) and one may have a fairly strong case for a dismissive attitude toward building corridors and stepping stones. We do not fully subscribe to this opinion. First of all, as we noted above, the conclusion may be valid only if we use the expected metapopulation extinction time as a measure of persistence, which is debatable on scientific and other grounds; a high patch occupancy may be politically more defensible than a long time to metapopulation extinction due to survival in only a few (perhaps distant) patches. Second, as the data shown in figure $1 D$ indicate, most metapopulations have low colonization probabilities, in which case matters are subtler. Third, such an opinion disregards the fact that increasing colonization is still beneficial, albeit not as beneficial as decreasing extinction in most cases, and nature managers may not always have the option to maintain a mainland or even diminish local extinction risk at all, especially in a very fragmented landscape. In that situation, they should have some idea whether they should, for example, improve some existing corridors or build new corridors. So, we think that our results should dim the enthusiasm for connectivity and restore the interest in local habitat management, but they should not lead to rejection of the metapopulation approach; we need to keep working at multiple scales.

We noted that figure $1 D$, with values of $(e, c)$ and $(e$, $c_{8}$ ) calculated from data, calls into question our first rule of thumb that we should focus on decreasing $e$ rather than on increasing $c$. However, the data may not represent classical metapopulations, and the models used in the calculations assume equal $e$ and $c$ across patches, which is obviously far from realistic. Moreover, recalling figure 2, we see, first, that if increasing $c$ is preferred, decreasing $e$ 
is not much less successful than increasing $c$ and, second, that if one chooses to increase $c$ based on incorrect information, we may be far from the optimal situation achieved by decreasing $e$. In sum, we feel that figure $1 D$ warns us to be cautious in using the rule of thumb but no more than that.

When we compared the effect of small changes in the probabilities (i.e., $\Delta e, \Delta c, \Delta e_{i}, \Delta c_{i j}$ ), we assumed that they were equal. Especially when comparing changes in $e$ and changes in $c$, we may be comparing apples and oranges; they only have in common that they are probabilities in the same way that apples and oranges are both fruit. A fair comparison seems possible only on the level of effort or, indeed, money. If we are to choose between putting a certain amount of effort or money $x$ on increasing $c$ or decreasing $e$, we need to know $\Delta e$ and $\Delta c$ as functions of $x$. A similar procedure should be followed with respect to comparisons between the $\Delta e_{i}^{\prime}$ s and between the $\Delta c_{i j}$ 's. Unfortunately, such a function is not easily formulated. One may try to relate the extinction and colonization probabilities to measurable quantities such as patch area $\left(A_{i}\right)$ and interpatch distance $\left(d_{i j}\right)$; see, for example, Gyllenberg and Silvestrov (1994), who put $e_{i}=\exp \left(-A_{i}\right)$ and $c_{i j}=$ $\exp \left[-A_{i} \exp \left(-d_{i j} / d_{c}\right)\right]$, with $d_{c}$ the characteristic dispersal distance, in a discrete-time model similar to the model of this article but without separation of extinction and colonization phases. Yet, even if area and interpatch distance are considered sufficient to describe extinction and colonization, these relationships are debatable. Moreover, we still require expressions of $\Delta A_{i}$ and $\Delta d_{i j}$ as functions of $x$. Therefore, we deliberately chose to stay on the level of extinction and colonization probabilities, thus avoiding these relatively arbitrary choices of the functional forms. And in fact, there is an alternative option that might incorporate some aspects of a function relating $\Delta e$ and $\Delta c$ to $x$ on this probability level: comparing transformed changes. For example, we could compare a change in the local extinction probability for all patches together with a change in the colonization probability for all connections together (i.e., all $n(n-1)$ pairs of patches); that is, we could require

$$
n \Delta e=n(n-1) \Delta c .
$$

Such a comparison will strengthen the effects of decreasing $e$ because $\Delta c$ will be smaller than $\Delta e$ for $n>2$. Another example is to compare relative changes in the parameters instead of absolute ones; that is, we could require

$$
\frac{\Delta e}{e}=\frac{\Delta c}{1-c},
$$

which is a mathematical formulation of the intuition that it will be more difficult to change $e$ when its value is small than when it is large and, analogously, that it will be more difficult to change $c$ when its value is close to 1 than when it is close to 0 . The consequence for figure $1 A-1 C$ is that all the lines lie a little higher, start higher for small $e$, and end on the $c=0$-axis at larger values of $e$, namely, at $e=1-(1 / n)$. So this choice would weaken the first rule of thumb, but the region in $(e, c)$-space where increasing $c$ is better than decreasing $e$ is still smaller than the remaining region. For the second and third rules of thumb, the requirement analogous to equation (20) becomes

$$
\begin{gathered}
\frac{\Delta e_{k}}{e_{k}}=\frac{\Delta e_{l}}{e_{l}}, \\
\frac{\Delta c_{i j}}{1-c_{i j}}=\frac{\Delta c_{k l}}{1-c_{k l}}
\end{gathered}
$$

for each $i, j, k$, and $l$. This may again have a weakening effect on the rules of thumb. Yet, as stated above, equation (20) is a mathematical formulation of an intuition, and it is questionable whether we should trust intuitions in this case. For example, well-connected patches may be much easier to connect than poorly connected patches precisely because of the fact that they are well connected: the infrastructure may allow for more connectivity. So, unless $\Delta e$ and $\Delta c$ as a function of $x$ are known, both equations (13) and (20) seem quite arbitrary, and we chose the simpler of the two.

Another feature of our analysis needs some attention here. All our randomly generated sets of probabilities were based on the uniform distribution, albeit on several different intervals. Ideally, we should have used extinction and colonization probabilities in real landscapes for real species, but if we knew these for so many different landscapes and species, most of this article would be superfluous. Since determining these probabilities is not an easy task, we are left to use some distribution of these probabilities that we think is fairly realistic. Yet, without strong arguments for a particular distribution, we chose the (arguably) simplest one: the uniform distribution. Encouraged by the limited effect of different intervals, we conjecture that different choices still support our conclusions but in a different degree.

In this article, we have only explored three of many questions that may be raised in management of metapopulations. There are many more combinations of changes (in particular, a combination of an increase in colonization probability and a decrease in extinction probability) than those we investigated; we tried to pick those that are both simple and useful. Also, we restricted our study to small changes in the parameters, although successive small changes or one large change could lead to different optima. 
Another interesting option would have been to study the effects of increasing the number of patches, but this discrete change is difficult to compare with small continuous changes in the probabilities (e.g., how to allocate colonization and extinction probabilities to a newly added patch). Furthermore, we examined some modifications of the main model, but many more are conceivable (multiple species, explicit local dynamics, catastrophes). Nevertheless, we believe that this article is a first step toward finding optimal strategies for nature management of metapopulations. The rules of thumb could act as guidelines or null hypotheses that need testing in particular cases and could steer further research, either with different (e.g., using graph and percolation theory; see, e.g., Keitt et al. 1997; Bunn et al. 2000; Urban and Keitt 2001) or more detailed models or, ideally, with empirical data. To our knowledge, no such model-based null hypotheses were available before. In addition, we feel that the processes underlying metapopulation dynamics have been somewhat disentangled and that new light has been shed on the complexity of the remaining entanglement.

\section{Acknowledgments}

We thank F. R. Adler, J. Grasman, F. van den Bosch, and J. Verboom for helpful comments on earlier versions of this article. We are also grateful to the anonymous reviewers and D. F. Doak for useful suggestions about the robustness and presentation of the results, which led to substantial improvement. Finally, we thank B. Engel for assisting in formulating the model for correlated extinctions.

\section{APPENDIX A}

\section{Derivation of Equations (3) and (4)}

In this appendix, we give the derivation of equations (3) and (4). Let us start with equation (3). The transition from $k$ to $l$ occupied patches can take place though $k-i$ extinctions to the intermediate state of $i$ occupied patches and, subsequently, through $l-i$ colonizations to the final state $l$ :

$$
P_{k l}=\sum_{i=0}^{\min (k, l)} p_{k i} p_{i l}
$$

where $i$ is bounded by the initial number of occupied patches $k$, on the one hand (no new occupied patches can arise after extinction), and by the final number of patches $l$, on the other (there can be no more patches after extinction than there can be after colonization). Assuming extinctions to be uncorrelated, extinction of $k-i$ patches has probability $e^{k-i}$. If $k-i$ patches go extinct, $i$ patches do not go extinct, which happens with probability $(1-e)^{i}$. There are

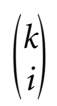

different ways in which $k-i$ patches can go extinct. Hence,

$$
p_{k i}=\left(\begin{array}{c}
k \\
i
\end{array}\right)(1-e)^{i} e^{k-i}
$$

Colonization of $l-i$ patches by the remaining $i$ patches has a probability of $1-$ (the probability of not being colonized by $i$ patches), that is, $\left[1-(1-c)^{i}\right]^{l-i}$. If $l$ patches are occupied in the end, then $n-l$ patches have not been colonized by the $i$ patches remaining after extinction, and this has probability $\left[(1-c)^{i}\right]^{n-l}$. There are

$$
\left(\begin{array}{l}
n-i \\
l-i
\end{array}\right)
$$

ways of $l-i$ empty patches being colonized if there are $n-i$ empty patches. Hence, 


$$
p_{i l}=\left(\begin{array}{c}
n-i \\
l-i
\end{array}\right)\left[1-(1-c)^{i}\right]^{l-i}\left[(1-c)^{i}\right]^{n-l}
$$

If we have correlated extinctions, then equation (A2) is no longer valid. Instead, we suppose that it becomes

$$
p_{k i}=\left(\begin{array}{c}
k \\
i
\end{array}\right) f(e, k, i, \rho)
$$

for some function $f$ that describes the probability of $k-i$ patches going extinct and $i$ patches not going extinct when extinctions are correlated with some measure of correlation $\rho$. To find an expression for $f$, we need knowledge of the mechanism that correlates the extinctions. Because this mechanism may be very complicated and our main goal is to get some insight in how any correlation in extinctions might change our results, we use a more phenomenological approach that yields an explicit expression for $f$.

We postulate that the extinction of each occupied patch $j$ is governed by a stochastic variable $y_{j}$ and that extinction occurs unless $y_{j}$ exceeds some critical or threshold value $y_{j}^{c}$. We assume, further, that each $y_{j}$ can be written as $y_{j}=$ $\mu_{j}+u+\varepsilon_{j}$, where $\mu_{j}$ is a constant and $u$ and $\varepsilon_{j}$ are normally distributed variables with zero mean and variances $\sigma^{2}$ and 1 , respectively. This entails that $y_{j}$ is also normally distributed with mean $\mu_{j}$ and variance $1+\sigma^{2}$. All $\mathrm{y}_{j}$ have $u$ in common, so $u$ (or equivalently $\sigma^{2}$ ) measures the strength of the correlation. To obtain a correlation measure with a value between 0 and 1 , we define $\rho=\sigma^{2} /\left(1+\sigma^{2}\right)$. Suppose we have $k$ occupied patches. From the construction of the $\mathrm{y}_{j}$, it follows that the vector $\boldsymbol{y}=\left(y_{1}, y_{2}, \ldots, y_{n}\right)$ is a $k$-dimensional normally distributed variable with mean vector $\boldsymbol{\mu}=\left(\mu_{1}, \mu_{2}, \ldots, \mu_{k}\right)$ and covariance matrix $\mathbf{Y}=\sigma^{2} \mathbf{J}+\mathbf{I}$, where $\mathbf{J}$ is a $k$-by- $k$ matrix with all elements equal to 1 and I is the $k$-by- $k$ identity matrix.

We can now relate the extinction probability $e_{j}$ to the variable $y_{j}$ in the following way:

$$
e_{j}=P\left(y_{j}<y_{j}^{\mathrm{c}}\right)=\Phi\left(\frac{y_{j}^{\mathrm{c}}-\mu_{j}}{\sqrt{1+\sigma^{2}}}\right) \Rightarrow y_{j}^{\mathrm{c}}-\mu_{j}=\sqrt{1+\sigma^{2}} \Phi^{-1}\left(e_{j}\right)
$$

where $\Phi(x)$ is the cumulative standard normal distribution in one dimension. Now we want to calculate the probability $f\left(e_{1} \ldots e_{k}, k, i, \rho\right)$ that certain patches go extinct while others do not. If we number the patches such that patches $1 \ldots k-i$ are the ones that go extinct and $k-i+1 \ldots k$ do not, this probability $f$ is given by

$$
\begin{aligned}
f\left(e_{1} \ldots e_{k}, k, i, \rho\right) & =P_{k}\left(y_{j}<y_{j}^{\mathrm{c}} \text { for } j \leq k-i \text { and } y_{j}>y_{j}^{\mathrm{c}} \text { for } j>k-i\right) \\
& =P_{k}\left(y_{j}-\mu_{j}<y_{j}^{\mathrm{c}}-\mu_{j} \text { for } j \leq k-i \text { and } y_{j}-\mu_{j}>y_{j}^{\mathrm{c}}-\mu_{j} \text { for } j>k-i\right) \\
& =\int_{-\infty}^{\left(1+\sigma^{2}\right)^{1 / 2} \Phi^{-1}\left(e_{1}\right)} \ldots \int_{-\infty}^{\left(1+\sigma^{2}\right)^{1 / 2} \Phi^{-1}\left(e_{k-i}\right)} \int_{\left(1+\sigma^{2}\right)^{1 / 2} \Phi^{-1}\left(e_{k-i+1}\right)}^{\infty} \ldots \int_{\left(1+\sigma^{2}\right)^{1 / 2} \Phi^{-1}\left(e_{k}\right)}^{\infty} N_{k}(\boldsymbol{y}, \overrightarrow{0}, \mathbf{Y}) d y,
\end{aligned}
$$

where we have attached the subscript $k$ to $P$ to denote that the probability concerns $k$ events, $N_{k}(\boldsymbol{y}, \overrightarrow{0}, \mathbf{Y})$ is the normal distribution in $k$ dimensions with zero mean and covariance matrix $\mathbf{Y}$, and we have used equation (A5). If we now substitute $e_{j}=e$ for all $j$ and use equation (A4) in equation (A1), we get the required equation (4).

We remark that to facilitate numerical calculation of the integral in equation (A6), one can use Ihm's (1959) formula, which in this case reads

$$
\int_{B} N_{k}(\boldsymbol{y}, \overrightarrow{0}, \mathbf{Y}) d \boldsymbol{y}=\frac{1}{\sigma \sqrt{2 \pi}} \int_{-\infty}^{\infty} e^{-(1 / 2)\left(z^{2} / \sigma^{2}\right)} \int_{B} N_{k}(\boldsymbol{y}-z \boldsymbol{d}, \overrightarrow{0}, \mathbf{I}) d y d z
$$

with $B$ the $k$-dimensional domain of integration and $\boldsymbol{d}$ a $k$-dimensional vector with all elements equal to 1 . Furthermore, one can use the fact that, since we have $e_{i}=e$, 


$$
P_{k}\left(y_{j}<y_{j}^{\mathrm{c}} \text { for } j \leq k-i \text { and } y_{j}>y_{j}^{c} \text { for } j>k-i\right)=\sum_{h=0}^{i}\left(\begin{array}{l}
i \\
h
\end{array}\right)(-1)^{h} P_{k-i+h}\left(y_{j}<y_{j}^{\mathrm{c}} \text { for } j \leq k-i+h\right),
$$

with, evidently, $P_{0}\left(y_{j}<y_{j}^{\mathrm{c}}\right.$ for $\left.j \leq 0\right)=1$ and $P_{1}\left(y_{j}<y_{j}^{\mathrm{c}}\right.$ for $\left.j \leq 1\right)=e$, which means that if there are $n$ patches, we only have to compute $n-1$ integrals to get all possible values $p_{k l}$.

\section{APPENDIX B}

\section{The Value of $e$ Satisfying Equation (12) and $c=0$}

In this appendix, we will show that the curves of figure $1 A$ and $1 B$ cross the $(c=0)$-axis for $e=1-1 /(n-1)$. First, we note from equations (5) and (10) that in the discrete-time case,

$$
\frac{\partial T_{\text {ext }}}{\partial x}=\frac{\partial}{\partial x}\left(\frac{1}{1-\lambda_{2}}\right)=\frac{1}{\left(\lambda_{2}-1\right)^{2}} \frac{\partial \lambda_{2}}{\partial x}
$$

and that in the continuous-time case,

$$
\frac{\partial T_{\mathrm{ext}}}{\partial x}=-\frac{\partial}{\partial x} \frac{1}{\lambda_{2}}=\frac{1}{\lambda_{2}^{2}} \frac{\partial \lambda_{2}}{\partial x}
$$

where $x$ denotes any one of the two parameters $e$ and $c$. Thus, we see that, in both cases, the equality

$$
\left.\frac{\partial T_{\mathrm{ext}}}{\partial e}\right|_{(e, c)}=-\left.\frac{\partial T_{\mathrm{ext}}}{\partial c}\right|_{(e, c)}
$$

is equivalent to the equality

$$
\left.\frac{\partial \lambda_{2}}{\partial e}\right|_{(e, c)}=-\left.\frac{\partial \lambda_{2}}{\partial c}\right|_{(e, c)}
$$

Below, we will use the following result from Caswell (1989): for a matrix A with elements $a_{i j}$, left eigenvector $\boldsymbol{w}$, right eigenvector $v$, and a parameter $x$, we have

$$
\frac{\partial \lambda_{2}}{\partial x}=\sum_{i} \sum_{j} \frac{\partial \lambda_{2}}{\partial a_{i j}} \frac{\partial a_{i j}}{\partial x}=\frac{1}{\boldsymbol{v} \cdot w} \sum_{i} \sum_{j} \bar{w}_{i} v_{j} \frac{\partial a_{i j}}{\partial x}
$$

where the dot represents the inner product and the bar represents the complex conjugate.

\section{Discrete Time}

For any $n$, when $c=0$, the second largest eigenvalue $\lambda_{2}$ of $P$ is $(1-e)$, and it has right eigenvector $\boldsymbol{v}=$ $(0,1, \ldots, k, \ldots, n)^{\mathrm{T}}$ if $e \neq 0,1(k=0,1, \ldots, n)$ and left eigenvector $\boldsymbol{w}=(-1,1,0, \ldots, 0)$. In this case, Caswell's formula gives 


$$
\begin{aligned}
& \left.\frac{\partial \lambda_{2}}{\partial x}\right|_{c=0}=\left.\frac{1}{\boldsymbol{v} \cdot \boldsymbol{w}} \sum_{i} \sum_{j} \bar{w}_{i} v_{j} \frac{\partial P_{i j}}{\partial x}\right|_{c=0} \\
& =\frac{1}{(0,1, \ldots, k, \ldots, n)^{\mathrm{T}} \cdot(-1,1,0, \ldots, 0)} \sum_{j=0}^{n}\left(-\left.v_{j} \frac{\partial P_{0 j}}{\partial x}\right|_{c=0}+\left.v_{j} \frac{\partial P_{1 j}}{\partial x}\right|_{c=0}\right) \\
& =\left.\sum_{j=1}^{n} j \frac{\partial P_{1 j}}{\partial x}\right|_{c=0}=\left.\frac{\partial}{\partial x} \sum_{j=1}^{n} j P_{1 j}\right|_{c=0} \\
& =\left.\frac{\partial}{\partial x} \sum_{j=1}^{n} \sum_{i=0}^{1}\left\{j\left(\begin{array}{l}
1 \\
i
\end{array}\right)(1-e)^{i} e^{1-i}\left(\begin{array}{c}
n-i \\
j-i
\end{array}\right)\left[1-(1-c)^{i}\right]^{j-i}\left[(1-c)^{i}\right]^{n-j}\right\}\right|_{c=0} \\
& =\left.\frac{\partial}{\partial x}\left[(1-e) \sum_{j=1}^{n}\left(\begin{array}{c}
n-1 \\
j-1
\end{array}\right) c^{j-1}(1-c)^{n-j} j\right]\right|_{c=0} \\
& =\left.\frac{\partial}{\partial x}\left[(1-e) \sum_{j=0}^{n-1}\left(\begin{array}{c}
n-1 \\
j
\end{array}\right) c^{j}(1-c)^{(n-1)-j}(j+1)\right]\right|_{c=0} \\
& =\left.\frac{\partial}{\partial x}(1-e)[1+(n-1) c]\right|_{c=0},
\end{aligned}
$$

where in the last line properties of the binomial distribution are used.

Now,

$$
\begin{aligned}
& \left.\frac{\partial \lambda_{2}}{\partial e}\right|_{c=0}=-\left.1[1+(n-1) c]\right|_{c=0}=-1, \\
& \left.\frac{\partial \lambda_{2}}{\partial c}\right|_{c=0}=\left.(1-e)(n-1)\right|_{c=0}=(1-e)(n-1),
\end{aligned}
$$

so condition (12) is satisfied in $c=0$ if

$$
e=1-\frac{1}{n-1}
$$

\section{Continuous Time}

When $c=0$, the second largest eigenvalue is $\lambda_{2}=-e_{r}$, which has right eigenvector $\boldsymbol{v}=(0,1, \ldots, k, \ldots, n)^{\mathrm{T}}$ if $e \neq$ $0,1(k=0,1, \ldots, n)$ and left eigenvector $\boldsymbol{w}=(-1,1,0, \ldots, 0)$. Again, we can compute the derivative of $\lambda_{2}$ with respect to a parameter $x\left(e_{r}\right.$ or $\left.c_{r}\right)$ in this case: 


$$
\left.\frac{\partial \lambda_{2}}{\partial x}\right|_{c=0}=\left.\frac{1}{\boldsymbol{v} \cdot \boldsymbol{w}} \sum_{i} \sum_{j} \bar{w}_{i} v_{j} \frac{\partial R_{i j}}{\partial x}\right|_{c=0}=\sum_{j=0}^{n}\left(\left.j \frac{\partial R_{1 j}}{\partial x}\right|_{c=0}\right)=\left.\frac{\partial}{\partial x} \sum_{j=0}^{n} j R_{1 j}\right|_{c=0}=\left.\frac{\partial}{\partial x}\left[-e_{r}+c_{r}(n-1)\right]\right|_{c=0} .
$$

First, we observe that from equations (11a) and (11b), it follows that

$$
\begin{aligned}
& \frac{d c_{r}}{d c}=\frac{d}{d c}[-\ln (1-c)]=\frac{1}{1-c}, \\
& \frac{d e_{r}}{d e}=\frac{d}{d e}[-\ln (1-e)]=\frac{1}{1-e} .
\end{aligned}
$$

Because

$$
\begin{aligned}
& \left.\frac{\partial \lambda_{2}}{\partial c}\right|_{c=0}=\left.\frac{\partial \lambda_{2}}{\partial c_{r}} \frac{d c_{r}}{d c}\right|_{c=0}=\left.\frac{1}{1-c} \frac{\partial}{\partial c_{r}}\left[-e_{r}+c_{r}(n-1)\right]\right|_{c=0}=n-1, \\
& \left.\frac{\partial \lambda_{2}}{\partial e}\right|_{c=0}=\left.\frac{\partial \lambda_{2}}{\partial e_{r}} \frac{d e_{r}}{d e}\right|_{c=0}=\left.\frac{1}{1-e} \frac{\partial}{\partial e_{r}}\left[-e_{r}+c_{r}(n-1)\right]\right|_{c=0}=-\frac{1}{1-e},
\end{aligned}
$$

equation (12) is satisfied in $c=0$ for $e=1-1 /(n-1)$.

\section{Literature Cited}

Akçakaya, H. R., and L. R. Ginzburg. 1991. Ecological risk analysis for single and multiple populations. Pages 73-85 in A. Seitz and V. Loeschcke, eds. Species conservation: a population-biological approach. Birkhäuser, Basel.

Allen, E. J., J. M. Harris, and L. J. S. Allen. 1992. Persistence-time models for use in viability analyses of vanishing species. Journal of Theoretical Biology 155:33-53.

Briers, R. A., and P. H. Warren. 2000. Population turnover and habitat dynamics in Notonecta (Hemiptera: Notonectidae) metapopulations. Oecologia (Berlin) 123: 216-222.

Brown, J. H., and A. Kodric-Brown. 1977. Turnover rate in insular biogeography: effect of immigration on extinction. Ecology 58:445-449.

Bunn, A. G., D. L. Urban, and T. H. Keitt. 2000. Landscape connectivity: a conservation application of graph theory. Journal of Environmental Management 59:265-278.

Burgman, M. A., S. Ferson, and H. R. Akçakaya. 1993. Risk assessment in conservation biology. Chapman \& Hall, London.

Caswell, H. 1989. Matrix population models. Sinauer, Sunderland, Mass.

Darroch, J. N., and E. Seneta. 1965. On quasi-stationary distributions in absorbing discrete-time finite Markov chains. Journal of Applied Probability 2:88-100.

Day, J. R., and H. P. Possingham. 1995. A stochastic metapopulation model with variability in patch size and position. Theoretical Population Biology 48:333-360.

Diekmann, O., and J. A. P. Heesterbeek. 2000. Mathematical epidemiology of infectious diseases: model building, analysis and interpretation. Wiley, Chichester.

Drechsler, M., and C. Wissel. 1998. Trade-offs between local and regional scale management of metapopulations. Biological Conservation 83:31-41.

Eber, S., and R. Brandl. 1994. Ecological and genetic spatial patterns of Urophora cardui (Diptera: Tephritidae) as evidence for population structure and biogeographical processes. Journal of Animal Ecology 63:187-199.

- 1996. Metapopulation dynamics of the tephritid fly Urophora cardui: an evaluation of incidence-function model assumptions with field data. Journal of Animal Ecology 65:621-630.

Etienne, R. S. 2000. Local populations of different sizes, mechanistic rescue effect and patch preference in the Levins metapopulation model. Bulletin of Mathematical Biology 62:943-958.

Etienne, R. S., and J. A. P. Heesterbeek. 2000. On optimal size and number of reserves for metapopulation persistence. Journal of Theoretical Biology 203:33-50. 
Frank, K., and C. Wissel. 1998. Spatial aspects of metapopulation survival: from model results to rules of thumb for landscape management. Landscape Ecology 13:363-379.

Gilpin, M., and B. L. Taylor. 1994. Reduced dimensional population transition matrices: extinction distributions from Markovian dynamics. Theoretical Population Biology 46:121-130.

Goel, N. S., and N. Richter-Dyn. 1974. Stochastic models in biology. Academic Press, New York.

Gosselin, F. 1998. Reconciling theoretical approaches to stochastic patch-occupancy metapopulation models. Bulletin of Mathematical Biology 60:955-971.

Gyllenberg, M., and D. S. Silvestrov. 1994. Quasi-stationary distributions of a stochastic metapopulation model. Journal of Mathematical Biology 33:35-70.

Halley, J. M., and Y. Iwasa. 1998. Extinction rate of a population under both demographic and environmental stochasticity. Theoretical Population Biology 53:1-15.

Hanski, I. 1994. A practical model of metapopulation dynamics. Journal of Animal Ecology 63:151-162.

Hanski, I., and O. Ovaskainen. 2000. The metapopulation capacity of a fragmented landscape. Nature 404:755-758.

Hanski, I., M. Kuussaari, and M. Nieminen. 1994. Metapopulation structure and migration in the butterfly Melitaea cinxia. Ecology 75:747-762.

Hansson, L. 1991. Dispersal and connectivity in metapopulations. Biological Journal of the Linnean Society 42:89-103.

Harrison, S., D. D. Murphy, and P. R. Ehrlich. 1988. Distribution of the bay checkerspot butterfly, Euphydryas editha bayensis: evidence for a metapopulation model. American Naturalist 132:360-382.

Hecnar, S. J., and R. T. M'Closkey. 1997. Spatial scale and determination of species status of the green frog. Conservation Biology 11:670-682.

Hill, J. K., C. D. Thomas, and O. T. Lewis. 1996. Effects of habitat patch size and isolation on dispersal by Hesperia comma butterflies: implications for metapopulation structure. Journal of Animal Ecology 65:725-735.

Ihm, P. 1959. Numerical evaluation of certain multivariate normal integrals. Sankhya 21:363-366.

Keilson, J. 1979. Markov chain models: rarity and exponentiality. Springer, Berlin.

Keitt, T. H., D. L. Urban, and B. T. Milne. 1997. Detecting critical scales in fragmented landscapes. Conservation Ecology (online) http://www.consecol.org/vol1/iss1/art4.

Kindvall, O., and I. Ahlén. 1992. Geometrical factors and metapopulation dynamics of the bush cricket, Metrioptera bicolor Philippi (Orthoptera: Tettigoniidae). Conservation Biology 6:520-529.

Klok, C., and A. M. de Roos. 1998. Effects of habitat size and quality on equilibrium density and extinction time on Sorex araneus populations. Journal of Animal Ecology 67:195-209.

Lei, G. C., and I. Hanski. 1998. Spatial dynamics of two competing specialist parasitoids in a host metapopulation. Journal of Animal Ecology 67:422-433.

Levins, R. 1969. Some demographic and genetic consequences of environmental heterogeneity for biological control. Bulletin of the Entomological Society of America 15:237-240.

- 1970. Extinction. Pages 75-107 in M. Gertenhaber, ed. Some mathematical problems in biology. American Mathematical Society, Providence, R.I.

Morrison, L. W. 1998. The spatiotemporal dynamics of insular ant metapopulations. Ecology 79:1135-1146.

Nürnberger, B. 1996. Local dynamics and dispersal in a structured population of the whirligig beetle Dineutus assimilis. Oecologia (Berlin) 106:325-336.

Sabelis, M., O. Diekmann, and V. A. A. Jansen. 1991. Metapopulation persistence despite local extinction: predator-prey patch models of the Lotka-Volterra type. Biological Journal of the Linnean Society 42:267-283.

Schultz, C. B. 1998. Dispersal behavior and its implications for reserve design in a rare Oregon butterfly. Conservation Biology 12:284-292.

Smith, A. T., and M. E. Gilpin. 1997. Spatially correlated dynamics in a pika metapopulation. Pages 407-428 in I. Hanski and M. E. Gilpin, eds. Metapopulation biology: ecology, genetics, and evolution. Academic Press, San Diego, Calif.

Sutcliffe, O. L., C. D. Thomas, and T. J. Yates. 1997. Correlated extinctions, colonizations and population fluctuations in a highly connected ringlet butterfly metapopulation. Oecologia (Berlin) 109:235-241.

Thomas, C. D., and S. Harrison. 1992. Spatial dynamics of a patchily distributed butterfly species. Journal of Animal Ecology 61:437-446.

Urban, D. L., and T. H. Keitt. 2001. Landscape connectivity: a graph-theoretic perspective. Ecology 82:1205-1218.

Van der Meijden, E., and C. A. M. Van der Veen-Van Wijk. 1997. Tritrophic metapopulation dynamics: a case study of ragwort, the cinnabar moth, and the parasitoid $\mathrm{Co}$ tesia popularis. Pages 387-405 in I. Hanski and M. E. Gilpin, eds. Metapopulation biology: ecology, genetics, and evolution. Academic Press, San Diego, Calif.

Verboom, J., J. A. J. Metz, and E. Meelis. 1993. Metapopulation models for impact assessment of fragmentation. Pages 172-191 in C. C. Vos and P. Opdam, eds. Landscape ecology of a stressed environment. IALE Studies in Landscape Ecology 1. Chapman \& Hall, London. Villard, M.-A., G. Merriam, and B. A. Maurer. 1995. Dy- 
namics in subdivided populations of neotropical migratory birds in a fragmented temperate forest. Ecology 76:27-40.

Vos, C. C., J. Verboom, P. F. M. Opdam, and C. J. F. ter Braak. 2001. Toward ecologically scaled landscape indices. American Naturalist 157:24-41.
Wilson, E. O., and E. O. Willis. 1975. Applied biogeography. Pages 523-534 in M. L. Cody and J. M. Diamond, eds. Ecology and evolution of communities. Harvard University Press, Cambridge, Mass.

Associate Editor: Daniel F. Doak 